\title{
Should Mathematics Education Be About Building Concrete Walls?
}

\author{
Fabien Paillusson ${ }^{1} \&$ Matthew Booth ${ }^{1}$ \\ ${ }^{1}$ School of Mathematics and physics, University of Lincoln, Lincoln LN6 7TS, UK \\ Corresponding author Matthew Booth: mbooth@lincoln.ac.uk
}

\begin{abstract}
Mathematics word problems have been used as a pedagogical tool for centuries and are now an enduring feature of contemporary mathematics education. However, a study by Gros, Sander, \& Thibaut in 2019 counterintuitively found that both expert and non-expert mathematicians are often unable to solve simple arithmetic equations when they are presented as word problems. The conclusions of this study were that the world semantics of the word problem affected the mathematical encoding of problems, especially for cardinal questions to do with the sizes of sets In this article we identify examples of apparently overlooked ambiguity in the word problems used in the aforementioned study, which appear to be more frequent in the cardinal problems, and which leads us to question the conclusions drawn by the authors. In the remainder of the article, we discuss more generally the ways in which linguistics and semantics may hinder the reduction of mathematics word problems to arithmetic equations and consider the pragmatics of studies which aim to assess mathematical ability using word problems.
\end{abstract}

\section{Introduction}

Mathematics is a discipline that involves the manipulation of abstract constructs while obeying clearly defined sets of rules. Mathematical results are those propositions about abstract mathematical objects that are obtained by a mathematics practitioner when correctly applying these rules. Failing to apply the rules correctly leads to invalid mathematical propositions.

\section{Arithmetic word problems}

Mathematical word problems (WPs) date back thousands of years yet their general form has remained remarkably consistent over the centuries and across cultures. The continued use of natural language in mathematics education despite the development of algebra is generally justified in two ways. First, WPs are thought to assist learners in making connections between the real world and mathematics by bringing the real world into classrooms (Krawitz, Schukajilow, \& Van Dooren, 2018). Second, it is claimed by some authors that WPs may be used to give concrete meaning to abstract mathematical objects (Greer, Verscahffel, \& De Corte, 2002).

However, Gerofsky has suggested that it is not possible for WPs to be equivalent to "real-world" scenarios since language is inherently ambiguous (Gerofsky, 2010). If mathematics itself may be treated as a written language with vocabulary, grammar and syntax, then the translation of mathematical problems into natural language may be tentatively subjected to pragmatic analysis from a translation studies perspective. Contemporary translation studies is heavily influenced by the functionalist approach, focusing on translation as a process or as a human action that ultimately serves a purpose (Kussmaul, 1995); when analysing the translation of a mathematics word problem into natural language, and vice versa, we cannot restrict discussion to notions of semantic equivalence but must also take into account the context. In other words, the purpose of 
the translation, the circumstances in which the problem is presented and the knowledge and expectations of the intended audience, must be considered.

In the education and application of mathematics a particularly interesting question arises: if a mathematics problem is framed in terms of real world situations and worded using a natural language, do advanced mathematics practitioners maintain a high rate of validity in their deduction of mathematical propositions and, if not, to what extent can the falsity of these propositions be attributed to an invalid mathematical reasoning?

In this article we claim that the multitude of factors that may affect an individual's interpretation of a mathematics WP, coupled with the inherent ambiguity of mathematics WPs, can result in multiple possible valid interpretations. Success with regards only to a single correct expected solution of a mathematics WP should therefore not be conflated with the concept of validity that is inherent to mathematical reasoning. A recent study (Gros, Sander, \& Thibaut, 2019) addressed this question and concluded that:

"[...] mathematically irrelevant contextual information may significantly hinder experts' abstract reasoning on problems within their very field of expertise, to the extent that they would not identify the validity of the solution handed out to them. Here, mathematical experts failed to do what they are good at: engaging in abstract reasoning on concrete entities to find a single-step solution"

This was then used as evidence to argue for the more general thesis that:

"This suggests that experts will never be completely freed from the influence of world knowledge; having an outstanding level in mathematics is not enough to systematically perceive that $14-2=12$."

We mostly disagree that these conclusions - which indeed contain some elements of insights - can be drawn from the study performed in (Gros et al., 2019) and shall explain why with reference to the broader context of the study. First, we shall briefly summarise its content.

\section{Summary of the Study by Gros et al.}

Gros and co-workers performed a study to assess to what extent formulating arithmetic problems as a WP would lead adults who are familiar with mathematics to misjudge the validity of a proposed answer. The methodology of the study consisted in providing adult subjects with a series of twelve elementary level mathematics WPs. Among the twelve problems, some were written in such a way that there was not enough data for the problem to be solved, while others had a very close phrasing but with enough data for the problem to be solved. Participants had then to state whether the provided answers were valid or if the problems were unsolvable. As far as we are aware, this was the sole data to be recorded alongside the time needed to answer each question. Two populations of adults were surveyed: normal adults having followed at least a few years of higher education in France and having definite familiarities with the relatively low level of mathematics required to solve the problems; and expert adults having successfully passed the entrance competition of the École Normale Supérieure in the sciences.

The study found that in both cohorts a substantial proportion (larger in normal adults than in expert adults) of participants would incorrectly tag a problem as being unsolvable when according to the authors it was solvable. It was also found that worded questions involving the estimation of the size of sets - termed cardinal problems in 
(Gros et al., 2019) - were much more prone to being incorrectly classified as unsolvable compared to so-called ordinal problems involving quantities such as lengths or durations. Furthermore, cardinal problems would require a larger amount of time for a correct answer to be given by the subjects. This lead Gros et al. to hypothesise that the world semantics of cardinal problems forced subjects to recode their mathematical representation of the problem, which takes a longer time than if the world semantics are congruent with the mathematical representation as was the case for the ordinal problems. This time-consuming recoding is then assumed to be more likely to lead to an incorrect representation of the mathematical content of the problem giving rise to incorrect claims of insolvability, even by experts. We shall now discuss whether or not these conclusions can really follow from the performed study.

\section{Analysis}

\section{From Natural Language to Formal Language}

All problems given to the participants were worded in a natural language. Solving the embedded mathematical problems necessitates the translation of words and situations into a formal expression of the form $z=x-y$ to which the standard rules of arithmetic apply. Since word problems contain redundant, non-mathematical information, we can suggest that the process of extracting the mathematically relevant content from a worded problem constitutes an operation of reduction. Under this definition, two differently worded problems are equivalent (or isomorphic to use the term from (Gros et al., 2019)) if their reduced (mathematical) form is the same. The claim, debatable in itself, that failing to solve a worded problem is equivalent to failing to solve the reduced mathematical problem can then only be made if the reduction of a worded problem into a mathematical one is unique. If that is not the case, then no conclusion can really be drawn, or at least they should be phrased much more carefully.

As it turns out, natural languages, as opposed to formal languages like mathematics, are notorious for being ambiguous (Gerofsky, 2010; Yuzhannikova, 2015). This may lead to non-unique reductions of certain worded problems. We will illustrate this point with one of the examples given in Table 2 of (Gros et al., 2019):

L1: Paul has a certain amount of red marbles.

L2: He also has blue marbles.

L3: In total, Paul has 14 marbles.

L4: Jolene has as many blue marbles as Paul, and some green marbles.

L5: She has two green marbles less than Paul has red marbles.

L6: How many marbles does Jolene have?

In this problem, if $z$ is the unknown total number of marbles that Jolene has, $x$ is the total number of marbles that Paul has and $y$ is the difference between the number of green marbles Jolene has and the number of red marble Paul has, then it is expected by Gros et al. that the answer is $z=x-y=14-2=12$. Is this answer unique? We do not believe so. A closer inspection of lines L1-3 simply tells us that a) Paul possesses red and blue marbles and b) in total he has 14 marbles. Nowhere is it stated that Paul possesses only blue and red marbles. For example, Paul could have green marbles whose existence in the world of the problem cannot be fathomed before L4. On that note L4-5 has a similar problem and simply tells us that Jolene possesses green and blue marbles without informing us that she only possesses these two colours. Thus, a logically minded person could legitimately conclude that there is not enough information to solve the problem; 
after all, if a worded problem needs to be univocally reduced to a mathematical one, it needs to be formulated precisely enough for this reduction not to rely on hidden preassumptions.

One could hypothesise that this type of linguistic ambiguity is more prominent in problems about sets - cardinal problems - since it becomes unclear whether the subsets described in the problem statements represent a complete covering of the full set, which is a necessary information if the participant is to determine the total number of elements in the set. If so, these ambiguities could explain the data reported in (Gros et al., 2019) without requiring the paradoxical phenomenon that "mathematically irrelevant contextual information may significantly hinder experts' abstract reasoning on problems within their very field of expertise, to the extent that they would not identify the validity of the solution handed out to them".

Even relatively simple cardinal problems can cause confusion. An illustrative (albeit anecdotal) example is the question "what is half of four trees". This question is clearly intended to be about the cardinality of sets, yet the context, i.e. the presence of the word tree, introduces some ambiguity to the intended meaning of the word half; it is not clear if half refers to number or for example the height of the trees.

While we do agree that worded problems such as the one analysed by Gros et al. can (and possibly should) confuse experts, the validity of their answers cannot be evaluated without knowing the reduced mathematical problem that the subjects came up with, even if possibly only at an intuitive level.

\section{The Context of the Study}

It could be argued that the point raised in the previous section about ambiguities is unfair since it is in fact easy to "see what is meant" in the worded questions and, in effect, these uncertainties should vanish in the context of elementary level arithmetic questions. Indeed, mathematical word problems have been described as a literary and pedagogical genre, the conventions of which students gradually become accustomed to (Gerofksy, 1996). Students tend to approach the "Word Problem Game" (De Corte, Verschaffel, \& de Win, 1985; DeFranco \& Curcio, 1997) with a willingness to suspend sense-making or provide unrealistic responses (Greer, 1997; Greer et al., 2002; Krawitz et al., 2018; Reusser \& Stebler, 1997; Schoenfeld, 1991; Sepeng, 2014).

A striking example is given by the question: "If a ship had 26 sheep and 10 goats onboard, how old is the ship's captain?" This question attracted much media attention recently as an unsolvable math problem. Indeed, at first it might appear to take the form of a mathematics word problem, yet on closer inspection it is clear that this question cannot be reduced to an arithmetic equation such as $z=x-y$. Regardless, the majority of students who were asked this question attempted to solve it as if it were a solvable mathematics word problem, that is, by trying to apply an arithmetic rule to the numerical values given in the question (Verschaffel \& De Corte, 1997). This phenomenon can be attributed to the so-called "didactical contract" pertaining to the "interpretation of the commitments, the expectations, the beliefs, the means, the results, and the penalties envisaged by one of the protagonists of a didactical situation (student, teacher, parents, society) for him or herself and for each of the others" (Brousseau, 1997).

Furthermore, according to the semantic features model (Littlefield \& Rieser, 1993) there are various ways in which students discriminate relevant information from irrelevant information in mathematics WPs. The experience level of students is important in this regard: less successful mathematics students tend to base judgements about the 
relevance of information on superficial aspects of the question structure, such as the order in which the information is presented (Cook, 2006; Cummins, 1991; Silver \& Smith, 1980; Suydam \& Weaver, 1980), while more successful mathematics students focused on higher order relations between the semantic categories in the WP text (Cook, 2006). Successful students first construct a mental model of the WP and then plan their strategy accordingly (Hegarty, Mayer, \& Monk, 1995; Thevenot, 2010). It was proposed by (Nathan, Kintsch \& Young, 1992) that two representational models are constructed, one qualitative and one quantitative, the former being a situational representation that is heavily influenced by the student's prior knowledge of the WP genre. It is this situational model that is used by students to make inferences when required.

While we may therefore assume that the ambiguity identified in the arithmetic word problems presented by Gros et al. (2019) would be negligible in a pedagogical context where pupils expect that there is a unique answer to the question, here the situation is somewhat different. Participants in the study were instructed to tell whether a given word problem is solvable (with a proposed solution being provided) or not. This might compel participants to closely examine the problem with a magnifying glass since they do not know at what level of logical details the authors of the study will evaluate their answers. Thus, we believe it is fairly plausible that given the context of the study, participants in general may have been even more sensitive to logical details than they would usually be. Furthermore, assuming that experts and non-experts differ in their expectations regarding the level of contextualisation in a mathematics question, then the two categories of participants would on the whole respond to the type of ambiguity outlined in the previous section in a different way.

A further complication that is not addressed in the discussion by Gros et al. is that participants were explicitly told that some questions were unsolvable due to a lack of information. A study by Park (2004) in which student participants were asked to solve anagrams, showed that simply informing participants that some of the anagrams would be unsolvable is enough to significantly hinder performance in terms of the number of anagrams solved as well as the time taken to complete the task. This calls into question the explanation provided by Gros et al. for the difference in response time of participants.

Since sensitivity to missing information increases with increased content specific knowledge (Low \& Over, 1989; Rehder, 1999), it is more likely that mathematics experts would notice (and assign relevance to) a lack of necessary information, especially when the instructions imply that this will be the case in some instances. Non-experts, on the other hand, are perhaps less experienced when it comes to the design and generation of questions and would not have such expectations; they are more likely to operate under the assumption that the translator is being cooperative.

\section{Discussion}

The results reported in (Gros et al., 2019) unambiguously show that adults, including experts, are confused by worded problems designed to lead them astray. Gros et al. equated this confusion with an inability to perform a simple arithmetic task or, more specifically, the inability to correctly assess the mathematical validity of a provided answer to an elementary mathematics problem. We believe this is not necessarily correct and have provided a possible alternative explanation for the results pertaining to cardinal problems, namely the ambiguity about whether or not the described subsets constitute a complete covering of the full set to be measured. This is not to say the interpretations by Gros et al. may not hold upon further studies; we simply claim that they cannot follow from the results reported in their study alone. 
The criticism formulated in this article can be addressed in future studies if issues inherent to linguistics are accounted for and analysed alongside the aspects more related to mathematics and its conceptualisation. Some possible avenues may be found in the literature on the education of mathematics where the phenomena reported by Gros et al. (2019) would be classified as misconceptions that even experts are not immunised against (Smith III, diSessa \& Roschelle, 1994). A first possibility would be to ask subjects to provide justifications for their answers or to explain their method, as in (Cook, 2006).

In addition, since mathematics ought to be unambiguous by definition, it appears desirable that worded problems aiming at contextualising mathematical problems be equally unambiguous or at least to as large an extent as possible. Grice's theory of cooperation (Grice, 1975) provides a descriptive framework of the various assumptions that may tacitly be assumed between two interlocutors efficiently communicating with each other, thereby underlying the "didactical contract" mentioned in the previous section. Indeed, Grice's maxims can be used as a theoretical framework to explain some of the cases reported above. For example, that pupils in examination conditions provide an arithmetic-based answer to the impossible problem of the captain's age implies that students assume that the question statement fulfil the maxims of quality and quantity, i.e. that it is not misleading and that it contains enough information to be solved.

By the same token, that students' performance is observed to worsen when they are told beforehand that some questions cannot be solved can be explained by conflicting maxims. If a pupil assumes that the received information about some questions being unsolvable are in accordance with the Gricean maxim of quality, i.e. that they are not intentionally misleading, then the WPs themselves will be approached under the expectation that the maxim of quantity is to be flouted in some instances. As a result, the cooperative principle cannot be applied consistently to both the instructions for the test and to individual questions. This uncertainty in the "didactical contract" to be abided by can explain the decrease in performance.

This latter example is particularly relevant to the study by Gros et al. (2019) which instructed the participants that some WPs were unsolvable and where participants had to endorse the validity of a provided solution or assert that it was unsolvable. Using Gricean maxims as a prescriptive framework, although not required, could help prevent the various linguistic ambiguities and the resulting mathematical uncertainties that we have reported in the previous section.

Finally, we wish to note that by putting an emphasis on having found WP formulations which constitute concrete walls for mathematicians, Gros et al. (2019) appear to endorse the arguable idea that finding WPs that most participants- including expert mathematicians - get wrong, is an objective to aim for in mathematics education. The very same study and results could be used to argue that there is a limit to which actual arithmetic problems can be embedded into WPs without losing their mathematical integrity; in which case the said WPs would stop serving their pedagogical and/or research purposes about the practice of mathematics. Rather than finding a limit to the aptitudes of mathematicians to do mathematics, Gros et al. have may well have just developed an assessment situation in which their suggested WPs are not about mathematics at all but about something else entirely.

\section{Conclusion}

In this article we have reviewed some of the difficulties stemming from linguistics, logic, semantics and pragmatics when WPs are used for the assessment and/or teaching of mathematical skills. 
By re-assessing the conclusions of a very recent research paper on WPs in light of these difficulties, we have also demonstrated that experts in mathematics education could draw invalid conclusions about the acquisition and expression of mathematical skills if linguistics and pragmatics are omitted from the analysis.

Finally, if WPs are to be used to foster mathematics skills in pupils, we have argued that it would be desirable to account for linguistics, logic and pragmatics when designing and assessing such WPs. Failing to do so could otherwise lead to the reverse effect whereby mathematics educators would effectively build a concrete wall between learners and mathematics itself.

\section{Disclosure statement}

No potential conflict of interest was reported by the authors.

\section{References}

(Brousseau, 1997) Brousseau, G. (1997). Theory of didactical situations in mathematics 1970-1990. Dordrecht: Kluwer.

(Cook, 2006) Cook, J. L. (2006). College Students and Algebra Story Problems: Strategies for Identifying Relevant Information. Reading Psychology, 27:2-3, 95-125

(Cummins, 1991) Cummins, D. D. (1991). Children's interpretations of arithmetic word-problems. Cognition and Instruction, 8, 261-289.

(De Corte, Verschaffel, \& de Win, 1985) De Corte, E., Verschaffel, L., \& de Win, L., (1985). Influence of rewording verbal problems on children's problem representations and solutions. Journal of Educational Psychology, 77(4), 460-470

(DeFranco \& Curcio, 1997) DeFranco, T. C., \& Curcio, F. R., (1997) A division problem with a remainder embedded across two contexts: Children's solutions in restrictive versus real-world settings. Focus on Learning Problems in Mathematics, 19(2), 58-72.

(Gerofksy, 1996) Gerofsky, S., (1996). A linguistic and narrative view of word problems in mathematics education. For the Learning of Mathematics, 16, 2

(Gerofsky, 2010) Gerofsky, S., (2010). The impossibility of 'real-life' word problems (according to Bakhtin, Lacan, Zizek and Baudrillard). Discourse: Studies in the Cultural Politics of Education, 31:1, 61-73

(Greer, 1997) Greer, B., (1997). Modelling reality in mathematics classrooms: The case of word problems. Learning and Instruction, 7, 293-307.

(Greer, Verscahffel, \& De Corte, 2002) Greer, B., Verschaffel, L., \& De Corte, E., (2002). "The Answer is Really 4.5": Beliefs About Word Problems in Beliefs: A Hidden Variable in Mathematics Education? Mathematics Education Library, vol 31. Springer, Dordrecht

(Grice, 1975) Grice, H. P., (1975). Logic and conversation (in P. Cole \& J. L. Morgan (Eds.)), Syntax and semantics: 3. Speech acts. New York: Academic Press.

(Gros, Sander, \& Thibaut, 2019) Gros, H., Sander, E., \& Thibaut, J., (2019). When masters of abstraction run into a concrete wall: Experts failing arithmetic word problems. Psychon Bull Rev, 26, 1738-1746 
(Hegarty, Mayer, \& Monk, 1995) Hegarty, M., Mayer, R. E., \& Monk, C. A., (1995). Comprehension of Arithmetic Word Problems: A Comparison of Successful and Unsuccessful Problem Solvers. Journal of Educational Psychology, Vol. 87, 1, 18-32.

(Krawitz, Schukajilow, \& Van Dooren, 2018) Krawitz, J., Schukajlow, S., \& Van Dooren, W., (2018). Unrealistic responses to realistic problems with missing information: what are important barriers? Educational Psychology, Vol. 38, NO. 10, $1221-1238$

(Kussmaul, 1995) Kussmaul, P., (1995). Training the Translator. Amsterdam: John Benjamins

(Littlefield \& Rieser, 1993) Littlefield, J., \& Rieser, J., (1993). Semantic Features of Similarity and Children's Strategies for Identifying Relevant Information in Mathematical Story Problems. Cognition and Instruction, 11(2), 133-188.

(Low \& Over, 1989) Low, R., \& Over, R., (1989). Detection of missing and irrelevant information within algebraic story problems. British Journal of Educational Psychology 59: 296-305.

(Nathan, Kintsch \& Young, 1992) Nathan, M. J., Kintsch, W., \& Young, E., (1992). A Theory of Algebra-Word-Problem Comprehension and Its Implications for the Design of Learning Environments. Cognition and Instruction, 9:4, 329-389

(Park, 2014) Park, S., (2014). Solvable or Not, That is the Question. PhD diss., Seoul National University, 2014.

(Rehder, 1999) Rehder, B., (1999). Detecting unsolvable algebra word problems. Journal of Educational Psychology, 91(4), 669-683.

(Reusser \& Stebler, 1997) Reusser, K., \& Stebler, R., (1997). Every word problem has a solution: The suspension of reality and sense-making in the culture of school mathematics. Learning and Instruction. 7. 309-328.

(Schoenfeld, 1991) Schoenfeld, A. H., (1991). On mathematics as sense-making: An informal attack on the unfortunate divorce of formal and informal mathematics. Informal reasoning and education (p. 311-343). Lawrence Erlbaum Associates, Inc.

(Sepeng, 2014) Sepeng, P., (2014). Use of Common-sense Knowledge, Language and Reality in Mathematical Word Problem Solving. African Journal of Research in Mathematics, Science and Technology Education, 18:1, 14-24.

(Silver \& Smith, 1980) Silver, E. A., \& Smith, J. P., (1980). Think of a related problem (S. Krulik \& R.E. Reys (eds.)), Problem Solving in School Mathematics, NCTM, Reston, Virginia, pp. 146-156.

(Smith III, diSessa \& Roschelle, 1994) Smith III, J. P., diSessa, \& A. A., Roschelle, J., (1994). Misconceptions Reconceived: A Constructivist Analysis of Knowledge in Transition. Journal of the Learning Sciences, 3:2, 115-163

(Suydam \& Weaver, 1980) Suydam, M., \& Weaver, J., (1980). Research on Mathematics Education Reported in 1979. Journal for Research in Mathematics Education, 11(4), 241-320

(Thevenot, 2010) Thevenot, C., (2010). Arithmetic word problem solving: Evidence for the construction of a mental model. Acta Psychologica, 133, 90-95 
(Verschaffel \& De Corte, 1997) Verschaffel, L., \& De Corte, E., (1997). Teaching Realistic Mathematical Modeling in the Elementary School: A Teaching Experiments with Fifth Graders. Journal for Research in Mathematics Education, 28, 577-601.

(Yuzhannikova, 2015) Yuzhannikova, M.A., (2015). A typology of linguistic ambiguity. Journal of Siberian Federal University, Humanities \& Social Sciences, 3 (8), 533-541. 